\title{
CHERN-SIMONS FORMS OF PSEUDO-RIEMANNIAN HOMOGENEITY ON THE OSCILLATOR GROUP
}

\author{
P. M. GADEA and J. A. OUBIÑA
}

Received 17 October 2002

\begin{abstract}
We consider forms of Chern-Simons type associated to homogeneous pseudoRiemannian structures. The corresponding secondary classes are a measure of the lack of a homogeneous pseudo-Riemannian space to be locally symmetric. In the present paper, we compute these forms for the oscillator group and the corresponding secondary classes of the compact quotients of this group.
\end{abstract}

2000 Mathematics Subject Classification: 57R20, 53C30, 53C50.

1. Introduction. The Chern-Simons theory supplies both in physics and differential geometry interesting characteristic forms and classes; for instance, it furnishes a mathematical formulation for some anomalies. The principle of the construction is a technique of comparison of connections. It is possible to give new secondary characteristic forms of Chern-Simons type for a pseudo-Riemannian manifold $(M, g)$ endowed with a homogeneous pseudoRiemannian structure $S$, by using the Levi-Civita connection $\nabla$ and the connection $\tilde{\nabla}=\nabla-S$. The tensor field $S$ satisfies Ambrose-Singer's equations (see (2.1)), and if the pseudo-Riemannian manifold $(M, g)$ is reductive homogeneous, then $\tilde{\nabla}$ is the canonical connection associated to a reductive decomposition.

This construction, explained in Section 2, furnishes odd-dimensional differential forms of degree greater than 1 , which are null if $S=0$. We recall that if $S=0$, then $(M, g)$ is pseudo-Riemannian locally symmetric. Under certain conditions, these forms are closed and define secondary classes.

The oscillator group is the only non-Abelian 4-dimensional simply connected solvable Lie group admitting a bi-invariant Lorentzian metric [7, 8]. Moreover, it is an example of homogeneous spacetime, which as a causal space satisfies the property of causal continuity [5, 6]. In [2], we considered the oscillator group equipped with its usual Lorentzian bi-invariant metric and we determined all the homogeneous Lorentzian structures on it and all the associated reductive pairs. We showed that there are six types of such pairs and proved that four of them are solutions of the Einstein-Yang-Mills equations. They have sources except for a particular case. In [4], we studied the homogeneous Lorentzian structures for a family of non-bi-invariant left-invariant metrics on the oscillator group. 
In the present paper, we calculate the forms of pseudo-Riemannian homogeneity on the oscillator group equipped with the above-mentioned leftinvariant metrics. Further, we obtain the corresponding secondary classes of the compact quotients of this group.

2. Chern-Simons forms associated to a homogeneous pseudo-Riemannian structure. Let $(M, g)$ be a connected $C^{\infty}$ pseudo-Riemannian manifold of dimension $n$ and signature $(k, n-k)$. Let $\nabla$ be the Levi-Civita connection of $g$ and $R$ its curvature tensor field. A homogeneous pseudo-Riemannian structure on $(M, g)$ is a tensor field $S$ of type $(1,2)$ on $M$ such that the connection $\tilde{\nabla}=\nabla-S$ satisfies

$$
\tilde{\nabla} g=0, \quad \tilde{\nabla} R=0, \quad \tilde{\nabla} S=0 .
$$

If $g$ is a Lorentzian metric $(k=1)$, we say that $S$ is a homogeneous Lorentzian structure. In [3], we proved that if $(M, g)$ is connected, simply connected, and geodesically complete, then it admits a homogeneous pseudo-Riemannian structure if and only if it is a reductive homogeneous pseudo-Riemannian manifold. This result extends the well-known characterization of Ambrose and Singer [1] of homogeneous connected, simply connected, and complete Riemannian manifolds in terms of homogeneous Riemannian structures.

Now, let $P=(P, M, G)$ be a principal bundle over the $n$-dimensional $C^{\infty}$ manifold $M$. Let $g^{r}(G)$ be the space of $\operatorname{Ad}(G)$-invariant polynomials of degree $r$. Let $D, \widetilde{D}$ be connections on $P$, with respective connection 1 -forms $\omega, \widetilde{\omega}$ and curvature forms $\Omega=d \omega+\omega \wedge \omega$ and $\widetilde{\Omega}$. If $I \in g^{r}(G)$, we can consider for each $r$ the $2 r$-form $I\left(\Omega^{r}\right)=I(\Omega, \ldots, \Omega)$ on $P$, which projects to a (unique) $2 r$ form on $M$, say again $I\left(\Omega^{r}\right)$, which is closed. Consider the connection given, for $t \in[0,1]$, by $\omega_{t}=\widetilde{\omega}+t(\omega-\widetilde{\omega})$, with curvature form $\Omega_{t}$. Then one has the transgression formula

$$
I\left(\Omega^{r}\right)-I\left(\widetilde{\Omega}^{r}\right)=d Q(\omega, \widetilde{\omega}),
$$

where

$$
Q(\omega, \widetilde{\omega}):=r \int_{0}^{1} I(\omega-\widetilde{\omega}, \underbrace{\Omega_{t}, \ldots, \Omega_{t}}_{r-1}) d t
$$

The Chern-Simons $(2 r-1)$-form $Q(\omega, \widetilde{\omega})$ on $M$ defines, if $I\left(\Omega^{r}\right)=I\left(\widetilde{\Omega}^{r}\right)$, a secondary class of $M$.

We consider the bundle of pseudo-orthonormal frames $p: \mathscr{O}_{k, n-k}(M) \rightarrow M$ with respect to the metric $g$ on $M$ of signature $(k, n-k)$. We define $\operatorname{Ad}(O(k, n-$ $k)$ ) -invariant polynomial functions $f_{1}, \ldots, f_{n}$ on the Lie algebra $\circ(k, n-k)$ by

$$
f(t, X)=\operatorname{det}(t I+X)=t^{n}+\sum_{r=1}^{n} f_{r}(X) t^{n-r}, \quad X \in \mathfrak{o}(k, n-k) .
$$


Let $\Omega$ be the curvature form of a connection $\omega$ in $0_{k, n-k}(M)$. Then, for each $f_{r}$, $r=1, \ldots, n$, there exists a unique closed $2 r$-form $v_{r}$ on $M$ such that $p^{*}\left(v_{r}\right)=$ $f_{r}(\Omega)$. One has $\operatorname{det}(I+\Omega)=p^{*}\left(1+v_{1}+\cdots+v_{n}\right)$; hence having characteristic forms $v_{r}$ of degree $2 r$, and a total form $\Upsilon\left(\mathbb{O}_{k, n-k}(M), \omega\right)=1+\sum_{r=1}^{n} v_{r}$. The forms $f_{r}(\Omega)$ are the elementary symmetric functions $s_{r}(\Omega), r=1, \ldots, n$, of the eigenvalues of $\Omega$ so that $\operatorname{det}(I+\Omega)=1+s_{1}(\Omega)+s_{2}(\Omega)+\cdots+s_{n}(\Omega)$. By using Newton's recursive formulas, one can further compute the functions $s_{r}(\Omega)$ in terms of the traces of the powers of $\Omega$ from the expressions

$$
\begin{aligned}
\operatorname{tr}\left(\Omega^{r}\right) & -s_{1}(\Omega) \operatorname{tr}\left(\Omega^{r-1}\right)+s_{2}(\Omega) \operatorname{tr}\left(\Omega^{r-2}\right)-\cdots \\
& +(-1)^{r-1} s_{r-1}(\Omega) \operatorname{tr}(\Omega)+(-1)^{r} r s_{r}(\Omega)=0, \quad r=1, \ldots, n,
\end{aligned}
$$

and since $\operatorname{tr} \Omega=0$, we have, after computation,

$$
\operatorname{det}(I+\Omega)=1-\frac{1}{2} \operatorname{tr}\left(\Omega^{2}\right)+\frac{1}{3} \operatorname{tr}\left(\Omega^{3}\right)+\frac{1}{4}\left(\frac{1}{2}\left(\operatorname{tr}(\Omega)^{2}\right)^{2}-\operatorname{tr}\left(\Omega^{4}\right)\right)+\cdots .
$$

If we consider here the Levi-Civita connection $\nabla$ and the linear connection $\tilde{\nabla}=\nabla-S$ with connection form $\widetilde{\omega}$ and curvature form $\widetilde{\Omega}$, where $S$ is a homogeneous pseudo-Riemannian structure on $(M, g)$, the general equation (2.2) can be written in this case as

$$
s_{r}(\Omega)-s_{r}(\widetilde{\Omega})=d Q(\omega, \widetilde{\omega})
$$

If $s_{r}(\Omega)=s_{r}(\widetilde{\Omega})$, then $Q(\omega, \widetilde{\omega})$ is closed, thus determining a secondary class. In particular, if $r=2$ or 3 , then this happens if $\operatorname{tr}\left(\Omega^{r}\right)=\operatorname{tr}\left(\tilde{\Omega}^{r}\right)$. We denote by $Q_{2 r-1}^{S}(M, g)$, or simply by $Q_{2 r-1}^{S}$, the form $Q(\omega, \widetilde{\omega})$ in (2.7).

DEFINITION 2.1. Let $(M, g)$ be a pseudo-Riemannian manifold and $S$ a homogeneous pseudo-Riemannian structure on $M$. The forms $Q_{2 r-1}^{S}(M, g)$, for each $3 \leq 2 r-1 \leq \operatorname{dim} M$, are called Chern-Simons forms of pseudo-Riemannian homogeneity (or simply forms of homogeneity) on $(M, g, S)$. The corresponding real cohomology classes $\left[Q_{2 r-1}^{S}\right](M, g)$ are called secondary classes of pseudoRiemannian homogeneity (or simply secondary classes of homogeneity).

The case $r=1$ in (2.7) is trivial. For $r=2$, one has the formula

$$
Q_{3}^{S}=-\frac{1}{2} \operatorname{tr}\left(2 \sigma \wedge \widetilde{\Omega}+\sigma \wedge d \sigma+2 \sigma \wedge \widetilde{\omega} \wedge \sigma+\frac{2}{3} \sigma \wedge \sigma \wedge \sigma\right)
$$

where $\sigma=\omega-\widetilde{\omega}$. One can obtain similar formulas for any $r$ with $2 r \leq \operatorname{dim} M$. We have the following proposition.

Proposition 2.2. If $S=0$, then $Q_{2 r-1}^{S}=0$ for each $r$.

Proof. The proof follows immediately from (2.3). 
3. Forms $Q_{3}^{S}$ on the oscillator group. The (4-dimensional) oscillator group is the simply connected Lie group Os with Lie algebra $05=\langle P, X, Y, Q\rangle$ having nonzero brackets

$$
[X, Y]=P, \quad[Q, X]=Y, \quad[Q, Y]=-X .
$$

3.1. The metrics $g_{\varepsilon}, \varepsilon \neq 0$. We first endow Os with the family of left-invariant Lorentzian metrics given at os by

$$
g_{\varepsilon}=\left(\begin{array}{llll}
\varepsilon & & & 1 \\
& 1 & & \\
& & 1 & \\
1 & & & \varepsilon
\end{array}\right), \quad-1<\varepsilon<1, \varepsilon \neq 0 .
$$

Let $\{\eta, \alpha, \beta, \xi\}$ denote the basis dual to $\{P, X, Y, Q\}$. Integrating Ambrose-Singer's equations (2.1), we obtain (see [4]) the family of homogeneous Lorentzian structures corresponding to $\left(\mathrm{Os}, g_{\varepsilon}\right)$ :

$$
\begin{aligned}
S_{(a, b)}^{\varepsilon}= & \frac{\varepsilon}{2} \beta \otimes(\eta \wedge \alpha)-\frac{\varepsilon}{2} \alpha \otimes(\eta \wedge \beta)-\frac{1}{2} \beta \otimes(\alpha \wedge \xi) \\
& +\frac{1}{2} \alpha \otimes(\beta \wedge \xi)+(a \eta+b \xi) \otimes(\alpha \wedge \beta), \quad a, b \in \mathbb{R} .
\end{aligned}
$$

We have

$$
\omega=\frac{1}{2}\left(\begin{array}{cccc}
0 & -\beta & \alpha & 0 \\
\varepsilon \beta & 0 & \varepsilon \eta-\xi & \beta \\
-\varepsilon \alpha & -\varepsilon \eta+\xi & 0 & -\alpha \\
0 & 0 & 0 & 0
\end{array}\right)
$$

Assuming that

$$
A=\left(\begin{array}{cccc}
0 & 0 & 0 & 0 \\
0 & 0 & -1 & 0 \\
0 & 1 & 0 & 0 \\
0 & 0 & 0 & 0
\end{array}\right)
$$

we can write

$$
\widetilde{\omega}=\left(\left(\frac{\varepsilon}{2}+a\right) \eta+\left(b-\frac{1}{2}\right) \xi\right) A, \quad \widetilde{\Omega}=\left(\frac{\varepsilon}{2}+a\right) \alpha \wedge \beta A .
$$

Then, after some calculations from (2.8), we obtain the following proposition.

Proposition 3.1. The form of homogeneity on the oscillator group (Os, $g_{\varepsilon}$ ) corresponding to the homogeneous Lorentzian structure $S_{(a, b)}^{\varepsilon}$ is given by

$$
\begin{aligned}
& Q_{3}^{S_{(a, b)}^{\varepsilon}}\left(\mathrm{Os}, g_{\varepsilon}\right) \\
& \quad=-\frac{1}{2}\left\{\left(\frac{\varepsilon^{2}}{2}-2 a(\varepsilon+a)\right) \eta \wedge \alpha \wedge \beta+\left(\frac{\varepsilon}{2}-2 b(\varepsilon+a)\right) \alpha \wedge \beta \wedge \xi\right\} .
\end{aligned}
$$


3.2. The metric $g_{0}$. This time we endow Os with the left-invariant Lorentzian metric

$$
g_{0}=\left(\begin{array}{llll} 
& & & 1 \\
& 1 & & \\
& & 1 & \\
1 & & &
\end{array}\right) \text {. }
$$

Then, integration of Ambrose-Singer's equations (2.1) gives us six families of homogeneous Lorentzian structures for $\left(\mathrm{Os}, g_{0}\right)$ [2]:

$$
\begin{aligned}
S_{(x, y, z, w)}= & \left(x \eta+x y \alpha+x z \beta+\left(w+\frac{1}{2}\right) \xi\right) \otimes(\alpha \wedge \beta) \\
& -\left(x z \eta+x y z \alpha+\left(x z^{2}+\frac{1}{2}\right) \beta+z w \xi\right) \otimes(\alpha \wedge \xi) \\
& +\left(x y \eta+\left(x y^{2}+\frac{1}{2}\right) \alpha+x y z \beta+y w \xi\right) \otimes(\beta \wedge \xi), \\
& x, y, z, w \in \mathbb{R}, x \neq 0 . \\
S_{(q, c)}= & \frac{1}{2} \xi \otimes(\alpha \wedge \beta)-\frac{1}{2} \beta \otimes(\alpha \wedge \xi)+(q \alpha+c \xi) \otimes(\beta \wedge \xi), \\
S_{(b, c)}= & \frac{1}{2} \xi \otimes(\alpha \wedge \beta)+\left(-\frac{1}{2} \beta+b \xi\right) \otimes(\alpha \wedge \xi) \\
& +\left(\frac{1}{2} \alpha+c \xi\right) \otimes(\beta \wedge \xi), \quad b, c \in \mathbb{R} . \\
& +\left(\left(\frac{1}{2}-\left(\frac{k^{2}}{t}\right)\right) \alpha-k \beta-\left(\frac{k b}{t}\right) \xi\right) \otimes(\beta \wedge \xi), \\
S_{(k, t, b)} & \frac{1}{2} \xi \otimes(\alpha \wedge \beta)+\left(k \alpha+\left(t-\frac{1}{2}\right) \beta+b \xi\right) \otimes(\alpha \wedge \xi) \\
& +\left(\frac{1}{2} \alpha+c \xi\right) \otimes(\beta \wedge \xi), \quad a, b, c \in \mathbb{R}, a \neq \frac{1}{2} . \\
S_{a}= & a(\xi \otimes(\alpha \wedge \beta)-\beta \otimes(\alpha \wedge \xi)+\alpha \otimes(\beta \wedge \xi)), a \in \mathbb{R}, a \neq \frac{1}{2} . \\
S_{(a, b, c)}= & a \xi \otimes(\alpha \wedge \beta)+\left(-\frac{1}{2} \beta+b \xi\right) \otimes(\alpha \wedge \xi) \\
&
\end{aligned}
$$

We have

$$
\omega=\frac{1}{2}\left(\begin{array}{cccc}
0 & -\beta & \alpha & 0 \\
0 & 0 & -\xi & \beta \\
0 & \xi & 0 & -\alpha \\
0 & 0 & 0 & 0
\end{array}\right)
$$


and for the first family (3.9), we get

$$
\begin{aligned}
& \widetilde{\omega}=(x \eta+x y \alpha+x z \beta+w \xi)\left(\begin{array}{cccc}
0 & z & -y & 0 \\
0 & 0 & 1 & -z \\
0 & -1 & 0 & y \\
0 & 0 & 0 & 0
\end{array}\right), \\
& \widetilde{\Omega}=d(x \eta+x y \alpha+x z \beta+w \xi)\left(\begin{array}{cccc}
0 & z & -y & 0 \\
0 & 0 & 1 & -z \\
0 & -1 & 0 & y \\
0 & 0 & 0 & 0
\end{array}\right),
\end{aligned}
$$

which lead to a nontrivial form $Q_{3}^{S_{(x, y, z, w)}}$. However, for the other five families of homogeneous Lorentzian structures, the corresponding matrices $\widetilde{\omega}$ and $\widetilde{\Omega}$ give

$$
\operatorname{tr}(\sigma \wedge \widetilde{\Omega})=\operatorname{tr}(\sigma \wedge d \sigma)=\operatorname{tr}(\sigma \wedge \widetilde{\omega} \wedge \sigma)=\operatorname{tr}(\sigma \wedge \sigma \wedge \sigma)=0
$$

and we have the following proposition.

Proposition 3.2. The forms of homogeneity on the oscillator group (Os, $g_{0}$ ) corresponding to the previous six families of homogeneous Lorentzian structures are null except for the first family $S_{(x, y, z, w)}$, for which

$$
\begin{aligned}
& Q_{3}^{S_{(x, y, z, w)}}\left(\mathrm{Os}, g_{0}\right)=-\frac{1}{2}\{ 2 x^{2}(-\eta \wedge \alpha \wedge \beta+z \eta \wedge \alpha \wedge \xi-y \eta \wedge \beta \wedge \xi) \\
&\left.-x\left(1+2 w+2 x\left(y^{2}+z^{2}\right)\right) \alpha \wedge \beta \wedge \xi\right\}
\end{aligned}
$$

3.3. Classes $\left[Q_{3}^{S}\right]$ of the compact quotients of Os. Now, we determine the secondary classes $\left[Q_{3}^{S}\right]$ of the compact quotients of the oscillator group. For this, we first note that given a left-invariant form $\alpha$ on a Lie group $G$, then it is invariant under the action of a discrete subgroup $\Gamma$ of $G$, so that there exists a form $\hat{\alpha}$ on the quotient $\Gamma \backslash G$ such that $\pi^{*}(\hat{\alpha})=\alpha$, where $\pi$ denotes the natural projection $\pi: G \rightarrow \Gamma \backslash G$. In the sequel, we will denote by $\hat{\alpha}$ such a projected form of a left-invariant form $\alpha$ on $G$ onto a compact quotient $\Gamma \backslash G$. If $g$ is a left-invariant metric on $G$, then it projects to a metric $\hat{g}$ on $\Gamma \backslash G$ such that the map $\pi:(G, g) \rightarrow(\Gamma \backslash G, \hat{g})$ is a local pseudo-Riemannian isometry. Moreover, the Levi-Civita connection $\nabla$ projects to the Levi-Civita connection $\hat{\nabla}$ on $\Gamma \backslash G$ and each homogeneous pseudo-Riemannian structure $S$ projects to a homogeneous pseudo-Riemannian structure $\hat{S}$ on $\Gamma \backslash G$, where $\Gamma$ is a uniform discrete subgroup of $G$. The computation of the Chevalley-Eilenberg cohomology of the oscillator algebra os gives us

$$
\begin{gathered}
H^{0}(\mathfrak{o s}, \mathbb{R})=\langle 1\rangle, \quad H^{1}(\mathfrak{o s}, \mathbb{R})=\langle[\xi]\rangle, \quad H^{2}(\mathfrak{o s}, \mathbb{R})=0, \\
H^{3}(\mathfrak{o s}, \mathbb{R})=\langle[\eta \wedge \alpha \wedge \beta]\rangle, \quad H^{4}(\mathfrak{o s}, \mathbb{R})=\langle[\eta \wedge \alpha \wedge \beta \wedge \xi]\rangle .
\end{gathered}
$$


The Lie algebra os is a semidirect product of the Heisenberg algebra $\mathfrak{h}_{3}$ and $\mathbb{R}$, and the oscillator group is a semidirect product Os $=\mathbb{R} \ltimes H_{3}$. If $\Gamma$ is a uniform discrete subgroup of the Heisenberg group $\mathrm{H}_{3}$, we can consider the solvmanifold $(\mathbb{Z} \ltimes \Gamma) \backslash$ Os. The cohomology of such solvmanifold is known to be isomorphic to that of $S^{1} \times S^{3}$ (see [9]); that is, $H^{k}($ os, $\mathbb{R}) \approx H^{k}((\mathbb{Z} \ltimes \Gamma) \backslash$ Os, $\mathbb{R})$ for each $k$. The forms project to the quotients and we have the following proposition.

Proposition 3.3. The form of homogeneity $Q_{3}^{S_{(a, b)}^{\varepsilon}}\left(\mathrm{Os}, g_{\varepsilon}\right)$ in Proposition 3.1 determines the secondary class $-(1 / 2)\left(\varepsilon^{2} / 2-2 a(\varepsilon+a)\right)[\hat{\eta} \wedge \hat{\alpha} \wedge \hat{\beta}]$ associated to the homogeneous pseudo-Riemannian structure $\hat{S}_{(a, b)}^{\varepsilon}$ induced on the compact quotient $\left((\mathbb{Z} \ltimes \Gamma) \backslash \mathrm{Os}, \hat{g}_{\varepsilon}\right)$ by the homogeneous pseudo-Riemannian structure $S_{(a, b)}^{\varepsilon}$ in (3.3).

Proposition 3.4. The form of homogeneity $Q_{3}^{S_{(x, y, z, w)}}\left(\mathrm{Os}, g_{0}\right)$ in Proposition 3.2 determines the secondary class $x^{2}[\hat{\eta} \wedge \hat{\alpha} \wedge \hat{\beta}]$ associated to the homogeneous pseudo-Riemannian structure $\hat{S}_{(x, y, z, w)}$ induced on the compact quotient ( $\mathbb{Z} \ltimes$ $\left.\Gamma) \backslash \mathrm{Os}, \hat{\mathfrak{g}}_{0}\right)$ by the homogeneous pseudo-Riemannian structure $S_{(x, y, z, w)}$ in (3.9).

REMARK 3.5. For the class of pseudo-Riemannian homogeneity in Proposition 3.3 , one has

$$
\left[Q_{3}^{S_{(a, b)}^{\varepsilon}}\right]\left((\mathbb{Z} \ltimes \Gamma) \backslash \text { Os, } \hat{g}_{\varepsilon}\right)=0, \quad \text { if } a=\frac{\varepsilon}{2}(-1 \pm \sqrt{2}) \text {. }
$$

Thus these two pseudo-Riemannian compact quotients of the oscillator group, endowed with those homogeneous pseudo-Riemannian structures are "more symmetric" than the spaces corresponding to the rest of values of $a$.

ACKNOWLEDGMENT. This work was partially supported by DGICYT (Spain) under Grant BFM2002-00141 and by Xunta de Galicia under Project PGIDT01PXI20704PR.

\section{REFERENCES}

[1] W. Ambrose and I. M. Singer, On homogeneous Riemannian manifolds, Duke Math. J. 25 (1958), 647-669.

[2] R. D. Díaz, P. M. Gadea, and J. A. Oubiña, Reductive decompositions and EinsteinYang-Mills equations associated to the oscillator group, J. Math. Phys. 40 (1999), no. 7, 3490-3498.

[3] P. M. Gadea and J. A. Oubiña, Homogeneous pseudo-Riemannian structures and homogeneous almost para-Hermitian structures, Houston J. Math. 18 (1992), no. 3, 449-465.

[4] - Homogeneous Lorentzian structures on the oscillator groups, Arch. Math. (Basel) 73 (1999), no. 4, 311-320.

[5] A. V. Levichev, Chronogeometry of an electromagnetic wave given by a biinvariant metric on the oscillator group, Siberian Math. J. 27 (1986), no. 2, 237-245.

[6]___ Methods of investigation of the causal structures of homogeneous Lorentz manifolds, Siberian Math. J. 31 (1990), no. 3, 395-408. 
[7] A. Medina, Groupes de Lie munis de métriques bi-invariantes [Lie groups with biinvariant metrics], Tôhoku Math. J. (2) 37 (1985), no. 4, 405-421 (French).

[8] A. Medina and P. Revoy, Les groupes oscillateurs et leurs réseaux [Oscillator groups and their lattices], Manuscripta Math. 52 (1985), no. 1-3, 81-95 (French).

[9] A. Tralle and J. Oprea, Symplectic Manifolds with no Kähler Structure, Lecture Notes in Mathematics, vol. 1661, Springer-Verlag, Berlin, 1997.

P. M. Gadea: Department of Mathematics, Institute of Mathematics and Fundamental Physics, Spanish Council for Scientific Research (CSIC), Serrano 144, 28006 Madrid, Spain

E-mail address: pmgadea@iec.csic.es

J. A. Oubiña: Departamento de Xeometría e Topoloxía, Facultade de Matemáticas, Universidade de Santiago de Compostela, 15782 Santiago de Compostela, Spain

E-mail address: oubina@zmat.usc.es 


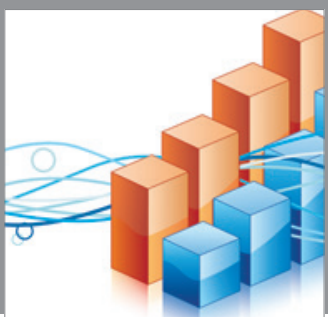

Advances in

Operations Research

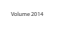

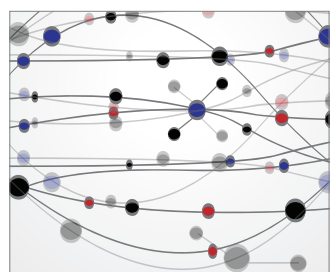

\section{The Scientific} World Journal
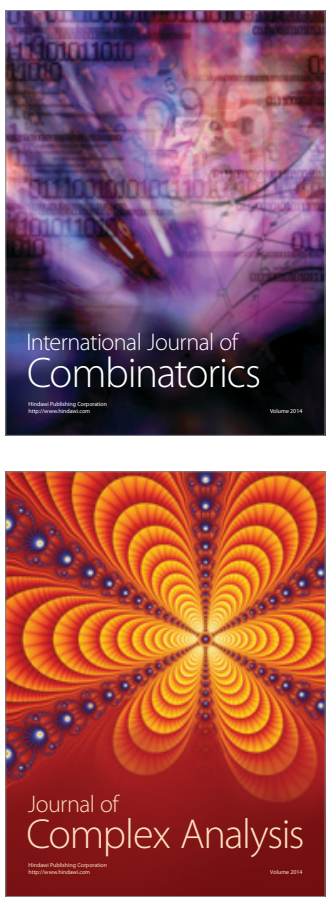

International Journal of

Mathematics and

Mathematical

Sciences
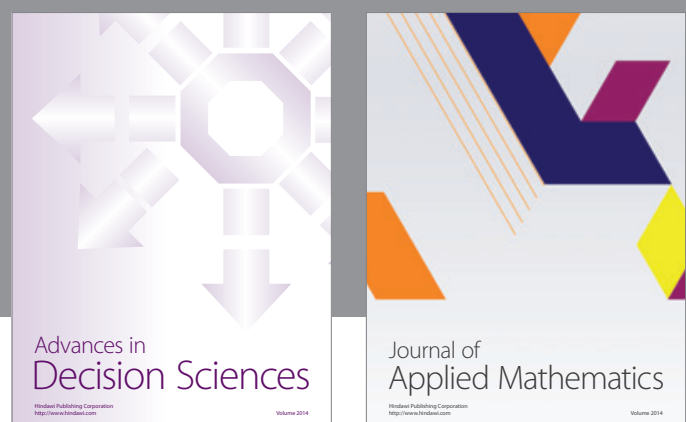

Journal of

Applied Mathematics
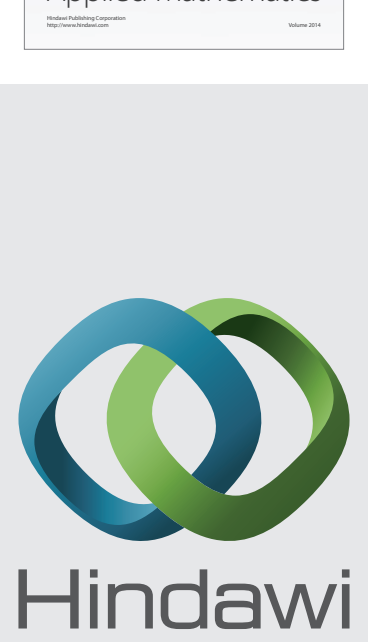

Submit your manuscripts at http://www.hindawi.com
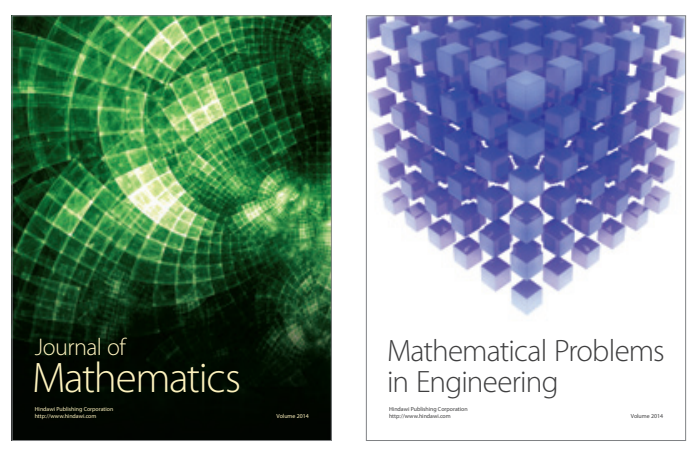

Mathematical Problems in Engineering
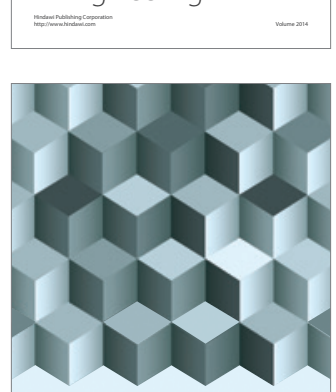

Journal of

Function Spaces
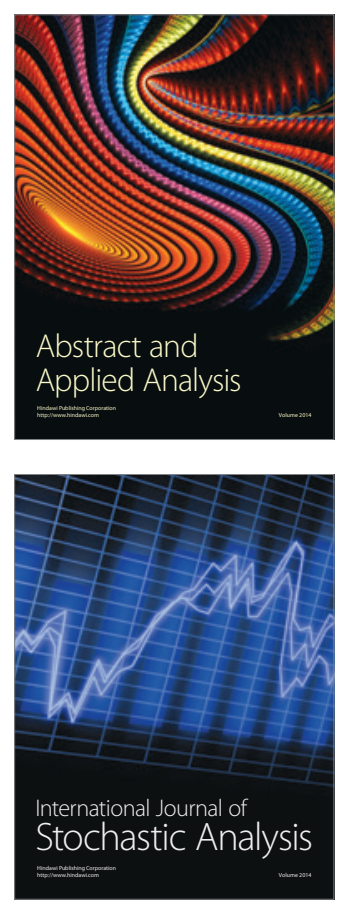

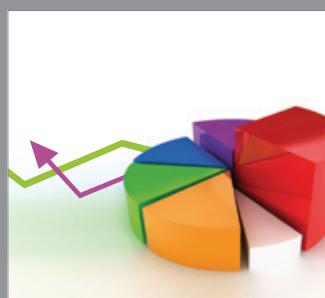

ournal of

Probability and Statistics

Promensencen
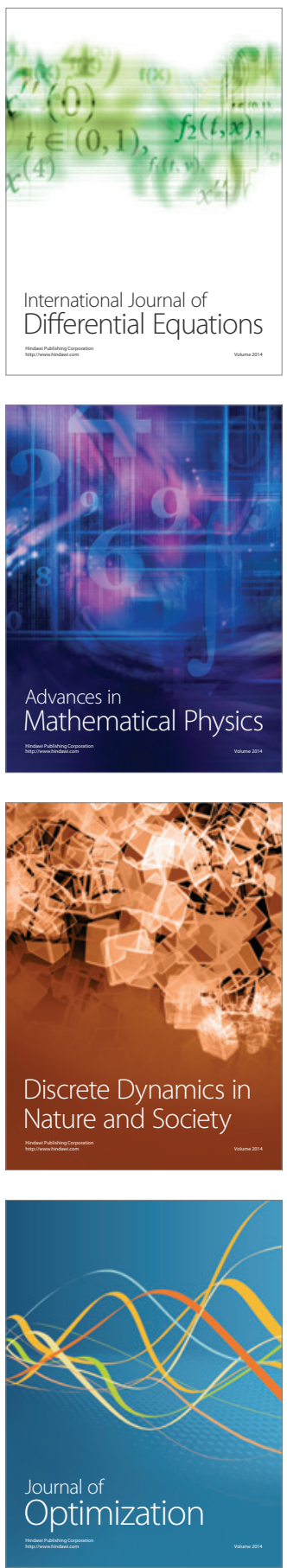